\title{
R Reseracth Sulure \\ Label Propagation Community Detection Algorithm Based on Density Peak Optimization
}

\author{
Yan Ma \\ Henan University \\ Guoqiang Chen ( $\nabla$ chengq08@163.com ) \\ Henan University
}

\section{Research Article}

Keywords: Community detection, label propagation, density peak

Posted Date: June 15th, 2021

DOl: https://doi.org/10.21203/rs.3.rs-607969/v1

License: (c) (i) This work is licensed under a Creative Commons Attribution 4.0 International License. Read Full License 


\title{
Label propagation community detection algorithm based on density peak optimization
}

\author{
Ma Yan, Chen Guoqiang* \\ School of Computer and Information Engineering, Henan University, 475004,Jinming \\ District,Kaifeng,Henan Province,China
}

Abstract-Community structure detection in complex network structure and function to understand network relations, found its evolution rule, monitoring and forecasting its evolution behavior has important theoretical significance, in the epidemic monitoring, network public opinion analysis, recommendation, advertising push and combat terrorism and safeguard national security has wide application prospect. Label propagation algorithm is one of the popular algorithms for community detection in recent years, the community detection algorithm based on tags spread the biggest advantage is the simple algorithm logic, relative to the module of optimization algorithm convergence speed is very fast, the clustering process without any optimization function, and the initialization before do not need to specify the number of complex network community. However, the algorithm has some problems such as unstable partitioning results and strong randomness. To solve this problem, this paper proposes an unsupervised label propagation community detection algorithm based on density peak. The proposed algorithm first introduces the density peak to find the clustering center, first determines the prototype of the community, and then fixes the number of communities and the clustering center of the complex network, and then uses the label propagation algorithm to detect the community, which improves the accuracy and robustness of community discovery, reduces the number of iterations, and accelerates the formation of the community. Finally, experiments on synthetic network and real network data sets are carried out with the proposed algorithm, and the results show that the proposed method has better performance.

Index Terms - Community detection, label propagation, density peak

\section{INTRODUCTION}

Community structure is a very important attribute in complex networks. Therefore, community structure not only plays a crucial role in the analysis of the social relations in human society [1], but also in the analysis of the functional relations between 
biological network organizations and organs [2], as well as the analysis of the citation relations between collaborative networks among scientists [3]. Therefore, the discovery of community structures from complex networks has been extensively studied in the past decade.

In 2002, Girvan and Newman achieved pioneering work pointing out that community structure is common in complex networks, and proposed modularity $\mathrm{Q}$ to measure the stability of communities in networks [1]. Although the definition of community structure has not been unanimously determined by clear relevant studies, it is conorolle concidered that a sommunity is a croun of nodes wrhich san also be ${ }^{*}$ Corresponding author. y tight

E-mail address: chengq08@163.com(Chen G.Q.)

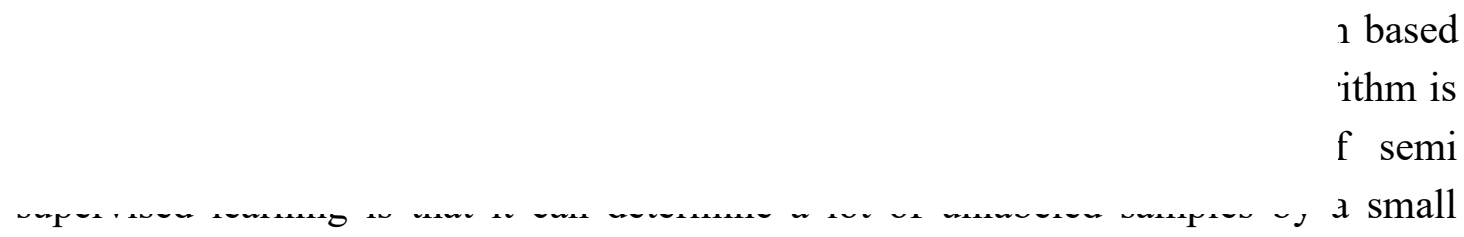

number of marked samples, thus improving the effectiveness of learning process [6-7] The basic idea of label propagation is to predict the label information of unlabeled nodes by using the topological relations between nodes from the label information of labeled nodes, and finally complete the division of the graph to form a clustering structure. Although this algorithm has the advantages of simple implementation, clear logic, no need to know the number of communities in advance, time complexity is close to linearity, etc., the unstable partition results and strong randomness are the defects of this algorithm. In each iteration of the label propagation algorithm, which community a node belongs to depends on the label with the largest cumulative weight of its neighbor nodes. Therefore, when more than one of the largest neighbor labels appears on a node, one of them will be randomly selected as its own label. This kind of randomness will bring avalanche effect, that is, a small clustering result error at the beginning will be continuously amplified. In addition, the updating order of node labels will also have a great impact. Obviously, the earlier the updating of the most important node will accelerate the process of convergence. In the label propagation algorithm, the closer the initial label is set to the core point, the more accurate the clustering effect is. However, in specific applications, it is often not feasible to know the number of communities in advance, and it is very inefficient to determine the number of communities $(\mathrm{K})$ by searching all possible candidate communities. Therefore, we are inspired by the density peak algorithm (DP) [8] and propose a label propagation algorithm based on density peak (DPLPA) for solving complex networks. The central idea of DP algorithm is that the core nodes are surrounded by other nodes in the same class, and there is no possibility for the core nodes to be closely connected. In other words, the core nodes have higher density, so this algorithm is feasible to 
calculate the core number. But unfortunately, DP algorithms cannot be directly used in complex network, so we to DP algorithm is improved, and it can be applied to a complex network, can be reasonably come to the core number, applied to the label propagation algorithm, according to the topology of the network that similarity matrix and priority to update nodes, reduce the randomness and the number of iterations.

\section{BACKGROUND}

\section{A. Label Propagation Algorithm}

Raghavan et al. proposed the label propagation algorithm (LPA) [9], which used the label values of a few preset nodes to divide the community structure on a large-scale complex network. However, the accuracy of LPA is low because of the randomness of propagation, which leads to a large error in clustering results. The reason is that when the neighbor node label frequency appears multiple highest values, the algorithm is fair to each label. Randomly select a label as the label of the update node. Therefore, the algorithm will appear small and fragmented communities or large communities which are not in line with the actual situation when the community is divided.The node propagation of the error is shown in Fig 1.
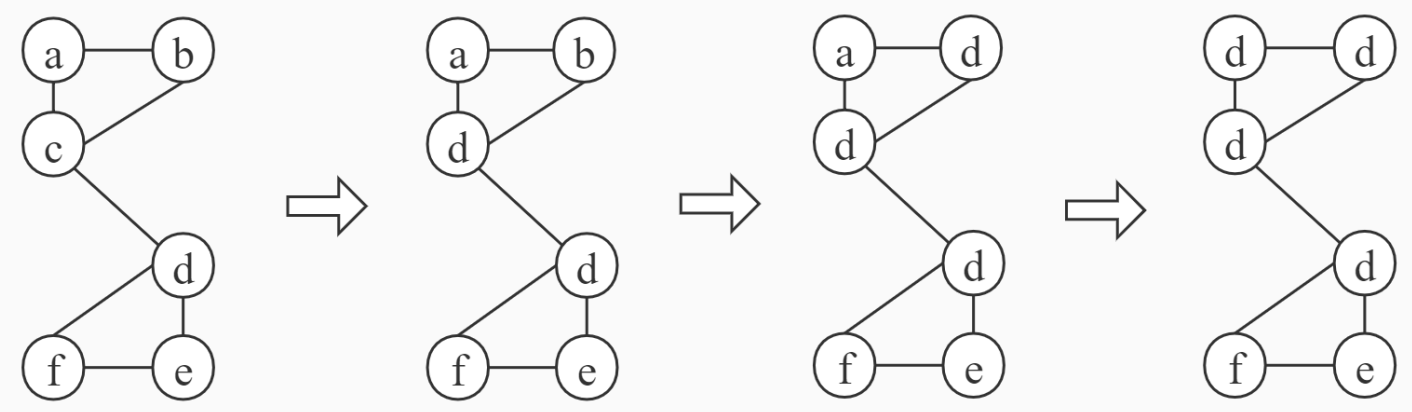

Fig. 1. Example of the propagation of an error

In view of the problems of LPA, domestic and foreign scholars have proposed many improvement measures. Tibély et al. [10] proved that the LPA will produce different community structures for the same network, and the algorithm still has a lot of randomness. Leung et al. [11] discovered the possibility of LPA application on tens of millions of networks, and found the potential of large-scale data application of the algorithm. Barber et al. [12] proposed the LPAm to solve the problem that the LPA cannot integrate different clustering results well by adding some restrictive conditions. Liu et al. [13] solved the problem that LPAm was easy to fall into local optimal solution by optimizing the modularity. Zhao et al. [14] calculated the $\mathrm{K}$ value by calculating the potential influence of nodes. When the $\mathrm{K}$ value is less than the actual 
number of communities, the algorithm will not get the correct partition result. Xie et al. [15] combined the label propagation algorithm with the Markov clustering algorithm (MCL) and proposed a new label propagation algorithm LabelRank. The biggest feature of the LabelRank algorithm is that a node can have multiple neighbor labels during the propagation process. Lin et al. [16] sorted the node weights and then updated the node labels in order. Zhang et al. [17] proposed a labeling algorithm based on edge clustering coefficient. Kipf et al. [18] extended the graph based label propagation algorithm and used graph convolution neural network for label propagation. The algorithm realized the propagation of label information through the aggregation of adjacent nodes. In addition, PageRank is used to quantify the importance of nodes, and LPAp algorithm [19] based on the importance of nodes is proposed. An improved community discovery algorithm based on feedback control [20], objective function [12], circle [21] and other methods for label propagation is proposed. The above algorithm is to optimize and improve the problem of node label in the propagation process, which can improve the stability and accuracy of LPA to a certain extent, but most of them bring more or less increased computational overhead, and do not achieve very ideal results.

However, Zhu et al. Proposed another label propagation algorithm (LP) in reference [22]. He described the clustering problem as a form of propagation on the graph, in which the label of one node propagates to the neighboring nodes according to the similarity between them. In this process, LP fixes a small number of tags on the known label data. Then, the tagged data, like a signal source, pushes the label through the unlabeled data. Therefore, an accurate number of known tags will play an important role in the propagation process of LP algorithm, greatly improving the accuracy of clustering results.

The algorithm based on label propagation can be described as follows:

1) Propagation label: $F=P \times F$

2) Reset the label of the core point in $F: F L=Y L$

3) Repeat steps 1) and 2) until $F$ converges

Where, Step 1) multiplies the probability transition matrix $P$ and the label matrix $F$ to propagate the label of each node to other nodes with the probability of $P$. If the similarity between two nodes is very high, the easier it is for each other's label to be replaced by its own. Step 2) the most important thing is the known label, which can't be changed, so every time it is propagated, it must return to the original label. As the label data point continues to propagate its label, the final class boundary passes through the high-density area and stays in the low-density interval. It is equivalent to the label node of each different category to divide the sphere of influence. 
However, it is still an open question to determine the number of known labels. Traditional community detection algorithm can obtain the number of communities by optimizing the objective function or evaluation index. However, these methods are easily affected by many factors such as initial matrix and optimization objective function, so it is difficult to accurately determine the number of communities. In order to solve the above problem, we use an improved density peak clustering to obtain the kernel number as the input parameter of LP.

\section{B. Density Peak Algorithm}

In 2014, Rodriguez et al. [8] proposed a density-based clustering method in Science, which can recognize clusters of various shapes, and the parameters are easy to determine. This method overcame the disadvantages of DBSCAN algorithm [23], which had large density differences among different classes and was difficult to determine the neighborhood range, and had strong robustness. The core idea of density peak algorithm (DP) is based on the assumption: for the center point of each cluster, the density of the cluster center point is greater than the density of surrounding neighbor points and the distance between the cluster center point and the higher density point is relatively large. Therefore, the DP algorithm has two quantities to calculate: the local density of the node and the distance from the high-density node. Usually, $\rho_{i}$ is used to represent the local density of node $i$, and $\delta_{i}$ is used to represent the distance between node $i$ and the high-density node.

There are two ways to define local density $\rho_{i}$, one of which is:

$$
\rho_{i}=\sum_{j} \chi\left(d(i, j)-d_{c}\right)
$$

where

$$
\chi(x)=\left\{\begin{array}{l}
1, \quad x \leq 0 \\
0, \text { otherwise }
\end{array}\right.
$$

Here $d(i, j)$ represents the distance from node $i$ to node $j$, and $d_{c}$ is the cut-off distance, that is, the number of nodes whose distance to node $i$ is less than $d_{c}$.

The second method is the Gaussian kernel function:

$$
p_{i}=\sum_{j} \exp \left(-\frac{d(i, j)^{2}}{d_{c}^{2}}\right)
$$

The minimum distance $\delta_{i}$ between node $i$ and other higher local density nodes is denoted by the formula defined as: 


$$
\delta_{i}=\left\{\begin{array}{l}
\max _{j}(d(i, j)), \text { otherwise } \\
\min _{j: \rho_{j}>\rho_{i}}(d(i, j)), \text { if } \exists \rho_{j}>\rho_{i}
\end{array}\right.
$$

When all the nodes have calculated $\rho_{i}$ and $\delta_{i}$, only the nodes with higher $\rho_{i}$ and $\delta_{i}$ can become the center points of the cluster, and the points with larger local density distance $\delta_{i}$ but smaller local density $\rho_{i}$ are abnormal points. The remaining nodes are assigned to the point with the highest local density among the neighbors.

Because the DP algorithm is a density-based clustering algorithm, it has the advantage of detecting clusters of arbitrary shape without the need to set the center point ( $\mathrm{K}$ value) in advance. Moreover, when selecting the center point, the selection process of the center point can be visually seen through the decision graph. However, DP algorithm still has some defects. Firstly, the value of cut-off distance $d_{c}$ needs to be set artificially, and improper setting will have a great impact on clustering results. Secondly, the central point needs to be selected artificially, so human subjective factors will affect the clustering results.

\section{METHODOLOGY}

In this section is introduced in this paper, the proposed DPLPA label propagation based on density peak optimization clustering algorithm. The core idea of DPLPA is to regard the high-density nodes surrounded by nodes of low-density neighbors as the community center points, and the distance between the community center points should be far away. In other words, a node with a higher density is more closely connected to its neighbors and is more likely to be the core point of the community. Community network is a kind of complex network with connections between nodes. However, DP algorithm is usually applied to connectionless network, and the distance between nodes should be calculated when calculating $\rho_{i}$ and $\delta_{i}$ indicators. If the distance between nodes in community network is calculated, the similarity between nodes will become meaningless because the distance between nodes is more uniform or even the same. Therefore, DP algorithm cannot be directly used to detect community networks. In order to solve this problem, this paper uses the improved DP algorithm [24] to obtain the number of communities in a complex network as the input parameter of the label propagation algorithm.

\section{A. Predictive Fetch of Label Matrix}

Let $G=(V, E)$ be a complex network with no direction and no weight. The node set $V$ contains $n$ nodes, the edge set $E$ contains $m$ edges, and the adjacency matrix of the graph $G$ is $A$. If node $i$ and node $j$ have an edge connected, then $a_{i j}=1$ in the adjacency matrix $A$ otherwise $a_{i j}=0$. Therefore, the node similarity formula of 
node $i$ and node $j$ is obtained, which is expressed by Salton index [25], also known as cosine similarity:

$$
S(i, j)=\frac{|N(i) \mathrm{I} N(j)|}{\sqrt{|| N(i)|\times| N(j)||}}
$$

Where $N(i)$ and $N(j)$ represent the neighbor nodes of node $i$ and node $j$, respectively, $|N(i)|$ represents the number of neighbor nodes of node $i$, so the molecular formula $|N(i) \mathrm{I} N(j)|$ represents the number of neighbors shared by node $i$ and node $j$, while denominator formula $\sqrt{|N(i)| \times|N(j)|}$ represents the number of neighbors expected to be shared by node $i$ and node $j$. The value of $S$ is between 0 and 1 . When $S$ is closer to 1 , the similarity between the two nodes is very high. The formula for the distance between node $i$ and node $j$ is as follows:

$$
d_{i, j}=\left\{\begin{array}{l}
\frac{1}{S(i, j)+\sigma}, \quad i \neq j \\
0, i=j
\end{array}\right.
$$

Among them $\sigma$ is a small positive number, in order to avoid the denominator being 0 .

Next, we have two methods to calculate the local density of the node, one is to use the Gaussian kernel function, the formula is as follows:

$$
\rho_{i}=\sum_{j} \exp \left(-\frac{d_{i, j}^{2}}{d_{c}^{2}}\right)
$$

Where $\rho_{i}$ represents the local density of node $i, d_{i, j}$ represents the distance between node $i$ and node $j, d_{c}$ represents the cut-off distance, and the size of $d_{c}$ is selected according to [8]. Then $\rho_{i}$ normalizes the value:

$$
\rho^{*}=\frac{\rho_{i}}{\max _{j} \rho_{j}}
$$

Then, start to define the distance formula between nodes:

$$
\delta_{i}=\left\{\begin{array}{l}
\max _{j}\left(d_{i j}\right), \text { if } \max \rho_{i} \\
\min _{j: \rho_{j>} \rho_{i}}\left(d_{i j}\right), \text { otherwise }
\end{array}\right.
$$

Among them, when the local density of node $i$ is the largest, its distance is the maximum value of the distance between node $i$ and other nodes. When the local 
density of node $i$ is not the maximum, its distance is the distance between the node whose local density is slightly larger than that of node $i$ and node $i$.

Then $\delta_{i}$ is standardized:

$$
\delta_{i}^{*}=\exp \left(-\left(\frac{d^{2}}{\delta_{i}^{2}}\right)\right)
$$

The threshold $d_{a}$ is selected from the list of $\delta$, which is about $80 \%$ of the list of $\delta$ from small to large [8].

Finally, take $\rho^{*}$ as the $X$-axis and $\delta^{*}$ as the $Y$-axis to generate a decision graph. Then calculate each node $\gamma=\rho^{*} \times \delta^{*}$, select a value greater than the sum of the average value of $\gamma$ and the standard deviation of $\gamma$ to enter the list, and then arrange them in order, and finally select the appropriate cut-off value as the core number (as the known label) and apply it to the label propagation algorithm.

\section{B. Label Propagation Algorithm Based on Density Peak}

LP is a graph-based clustering algorithm, so need to construct a graph first. The nodes of the graph are the data points. This paper uses the Gaussian kernel method to construct the weight between the two nodes:

$$
w_{i j}=\exp \left(-\frac{d_{i j}^{2}}{\beta^{2}}\right)
$$

Among them, $d_{i j}$ is the distance between node $i$ and node $j$, and $\beta$ is the hyper-parameter, and the similarity matrix composed of weight $w$ is obtained.

Next, the known label is propagated through the edges between nodes. The greater the weight of the edge, the more similar the two nodes, and the easier the label is to spread. Define the probability transition matrix:

$$
P_{i j}=P(i \rightarrow j)=\frac{w_{i j}}{\sum_{k=1}^{n} w_{i k}}
$$

Where $P_{i j}$ represents the probability of propagating the label of node $i$ to node $j$. Since there are known $K$ core points with known labels, a $K \times K$ label matrix $Y L$ is defined. The $i$ th row represents the label indication vector of node $i$, that is, if the label of the $i$ th node is $i$, then The $i$ th element is 1 , and the rest are 0 . It also defines an unlabeled matrix $Y U$ of unlabeled nodes. Combine to get the label matrix of all nodes: 


$$
F=[Y L, Y U]
$$

Then, the label matrix $F$ is propagated according to the similarity between nodes in the probability matrix $P$, the formula is as follows:

$$
F=P F
$$

After the propagation, the $Y L$ in the known label matrix $F$ changes during the propagation process, but $Y L$ is our previous predictive fetched, which is accurate and the label should not be changed. Therefore, need to reset the label matrix $F$, and the formula is as follows:

$$
F L=Y L
$$

Then, the label matrix $F$ is propagated, reset, and iterated until the change difference of unlabeled labels in $F$ reaches the critical point. At this moment, DPLPA completes the label partition. Table 1 shows the algorithm flow of DPLPA.

Table1 gives the pseudo-code of DPLPA.

\section{Algorithm 1 DPLPA}

Input: $G=(V, E)$

Output: A clustering for $G$

1 : Construct adjacency matrix $A$ from complex network $G=(V, E)$.

2 : Calculate node similarity $S$ by Eq. (5).

3 : Calculate the distance matrix $d$ between nodes by Eq. (6).

4 : Calculate the local density of the node $\rho^{*}$ by Eq.(7)、(8).

5 : Calculate $\delta^{*}$ by Eq. (9) 、(10).

6 : Calculate $\gamma=\rho^{*} \times \delta^{*}$ get $K$ core points.

7 : Get probability transition matrix $P$ by Eq. (11) 、(12).

8 : Build label matrix $F$ by Eq. (13).

9: while $F$ convergence criteria not reached do

10: $\quad F=P F$

11: $\quad F L=Y L$

\section{2: end while}

13: /* Iteratively update $F$ until convergence, and the label change of the node has 
been very small. $* /$

After obtaining the clustered label matrix $F$, the algorithm will gather the nodes with the value of 1 in the same dimension from $F$ together to form a community. All nodes are divided according to the dimension. The clustering algorithm is finished and the complex network is also divided.

\section{EXPERIMENTAL STUDY}

In order to assess our algorithm, we use a variety of real and synthetic data sets to test, and some classic methods to compare at the same time, including: DPLPA algorithm in this paper, Newman fast greedy algorithm $(F N)$ [26], Louvain algorithm $(B G L L)$ [27], LPA [9], improved label propagation algorithm (LPAm) [12]. The hardware environment of the experiment is as follows: Inter(R)Core(TM)i7-7700M CPU, 3.60GHz and 8GB memory. The DPLPA algorithm is implemented in Python3.7 64-bit.

\section{A. Evaluation Metrics}

In this article, in order to verify the accuracy of the algorithm, we use the community discovery modularity function $Q$ [26] proposed by Newman as the evaluation index of the experiment. Modularity is defined as:

$$
Q=\frac{1}{2 E} \sum_{i j}\left[A_{i j}-\frac{k_{i} k_{j}}{2 E}\right] \theta\left(c_{i}, c_{j}\right)
$$

Where $E$ represents the total number of edges of the community network, $A$ represents the adjacency matrix, $k_{i}$ represents the degree of node $i$, and $c_{i}$ represents the community allocated by node $i . \theta\left(c_{i}, c_{j}\right)$ is defined as follows:

$$
\theta\left(c_{i}, c_{j}\right)= \begin{cases}1, & (i, j) \in c, \\ 0, & \text { otherwise }\end{cases}
$$


Among them, when node $i$ and node $j$ are in the same community, $\theta\left(c_{i}, c_{j}\right)$ is 1 , otherwise it is 0 .

At the same time, we still use standardized mutual information ( NMI ) [28] to measure the similarity of two clustering results. It is an important measure of community discovery. It can basically objectively evaluate the comparison between a community division and a real division. Accuracy. The value range of $N M I$ is [0,1], and the higher the value, the closer the divided community is to the real community result. $N M I$ is defined as:

$$
\operatorname{NMI}(A, B)=\frac{-2 \sum_{i=1}^{C A} \sum_{i=1}^{C B} C_{i j} \log \left(C_{i j} N / C_{i .} C_{. j}\right)}{\sum_{i=1}^{C A} C_{i .} \log \left(\frac{C_{i .}}{N}\right)+\sum_{j=1}^{C B} C_{. j} \log \left(\frac{C_{. j}}{N}\right)}
$$

Among them, $A(B)$ represents the community discovery algorithm $A(B), C$ is the confusion matrix, $C_{i j}$ represents the number of nodes shared in the method $A(B)$ partition, $C A(C B)$ represents the number of communities in the community discovery method $A(B)$, and $C_{i .}\left(C_{. j}\right)$ represents the $i$-th row (column $j$ ) in $C$ and $N$ represents the number of nodes. If the clustering results of methods $A$ and $B$ are the same, then $\operatorname{NMI}(A, B)=1$.

\section{B. Performance on Synthetic Networks}

The use of artificially synthesized networks to evaluate the effectiveness of the algorithm has become an effective means to test the pros and cons of the algorithm. Among them, the most used Benchmark test network for community detection, LFR Benchmark, was proposed by Lancichineti Andrea [29]. The LFR reference network is an extension of the GN reference network [1] and has high practical value. The LFR benchmark network reflects the heterogeneity of community distribution and the power-law distribution of node degrees. Some of the important parameters are described as follows: $n$ represents the number of nodes, $k$ represents the average degree of nodes, max $k$ represents the maximum degree of nodes, and min $c$ represents the minimum community size, $\max c$ represents the maximum community size, $\tau 1$ and $\tau 2$ represent the negative exponents of the power-law distribution of node degree and community size respectively, $\mu$ is equal to the ratio of the number of connected edges between communities in the network to the total number of edges, to express the obvious degree of the community in the network, the smaller the $\mu$ value, the more obvious the structure of the community. Fig. 2 shows the comparison of the algorithm's NMI experiment results on the LFR benchmark data set. 


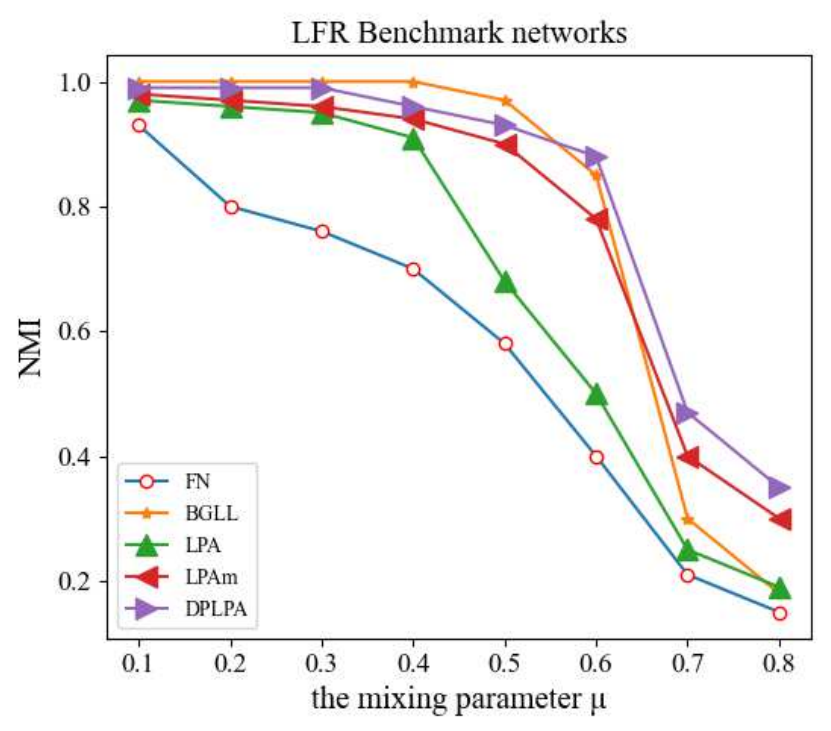

Fig. 2 Experimental results on benchmark data set

The parameters set in this LFR experiment are: $n=1000, k=15, \max k=40$, $\min c=20, \max c=50, \tau 1=2, \tau 2=1$, the range of $\mu$ is from 0.1 to 0.8 . It can be seen from fig 2 that when $\mu$ is small, that is, the community structure of the complex network is obvious, the $N M I$ values of the other algorithm results are high except for the FN algorithm. The $N M I$ value of the FN algorithm and the LPA both began to decrease significantly. The remaining algorithms all began to decrease when the $\mu$ value was 0.6 , but the DPLAP decreased relatively slowly compared with the BGLL and LPAm, and finally the NMI the value is higher, so this can indicate that the DPLPA has a higher accuracy rate in community exploration and has better stability in high-complexity community exploration.

\section{Real-World Networks}

In order to further compare the pros and cons of the algorithms, this paper also tested the algorithm in a few real social networks. These networks are of different sizes but are representative and involve various fields. See table 2 for details, where $n$ represents the node, $m$ represents the number of edges, and $k$ represents the number of communities that have been identified.

Table 2 Concrete description of real network

\begin{tabular}{ccccc}
\hline Network & $n$ & $m$ & $k$ & Descriptions \\
\hline Karate & 34 & 78 & 2 & Zachary's karate club
\end{tabular}




$\begin{array}{lcccc}\text { Dolphins } & 62 & 159 & 2 & \text { Dolphin social network } \\ \text { Polbook } & 105 & 441 & 3 & \text { Books about US politics } \\ \text { Football } & 115 & 616 & 12 & \text { American college football }\end{array}$

Among them, Karate [30] is a data set of member relations of a university karate club in the United States, which is constructed based on the interactions between club members and is often used in the analysis of social networks. Dolphins [31] is a member network constructed from the living habits of 62 wide-mouthed dolphins, and the dolphins that are often together correspond to an edge between nodes. Polbook [32] is a community network constructed through political books sold on Amazon in the United States. Each node represents a book. If two books are purchased by the same customer, there is an edge between them on the corresponding node. Football [1] is a network constructed by the American college football schedule. The nodes represent the participating teams. If there is a match between them, there will be an edge between the nodes. The calculation results of different algorithms on different networks are shown in table 3 and fig 3.

Table $3 \mathrm{Q}$ value comparison of different algorithms in real network

\begin{tabular}{cccccc}
\hline Networks & FN & BGLL & LPA & LPAm & DPLPA \\
\hline Karate & 0.3807 & 0.4188 & 0.3450 & 0.3496 & 0.3714 \\
Dolphins & 0.4955 & 0.5188 & 0.4788 & 0.4913 & 0.3789 \\
Polbook & 0.5020 & 0.4986 & 0.4953 & 0.4888 & 0.5063 \\
Football & 0.5497 & 0.6046 & 0.5445 & 0.5780 & 0.5539 \\
\hline
\end{tabular}




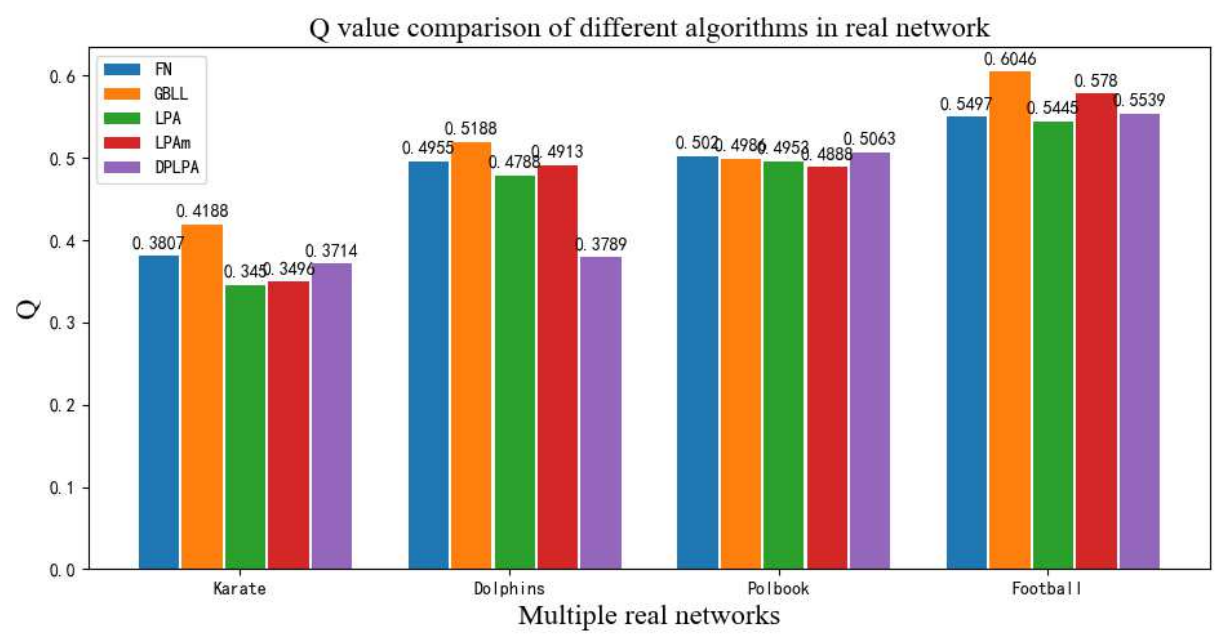

Fig. 3 Modularity Q comparison of multiple algorithms on multiple data sets

In order to better compare the clustering effect of DPLPA on the data set, this paper takes the Football data set to make a detailed explanation. The actual grouping of Football data set is shown in table 4, and the clustering effect of DPLPA is shown in fig 4.

Table 4 Network actual grouping of football data sets

\begin{tabular}{cc}
\hline Groups & Numbers \\
\hline 1 & 22634384690104106110 \\
2 & 203036568095102 \\
3 & 3714163340486165101107 \\
4 & 461141537375828599103108 \\
5 & 4549586776879293111113 \\
6 & 3743818391 \\
8 & 131519273235394455627286100 \\
9 & 151017244294105 \\
10 & 89222352697879109112 \\
11 & 18212857636671778896114
\end{tabular}




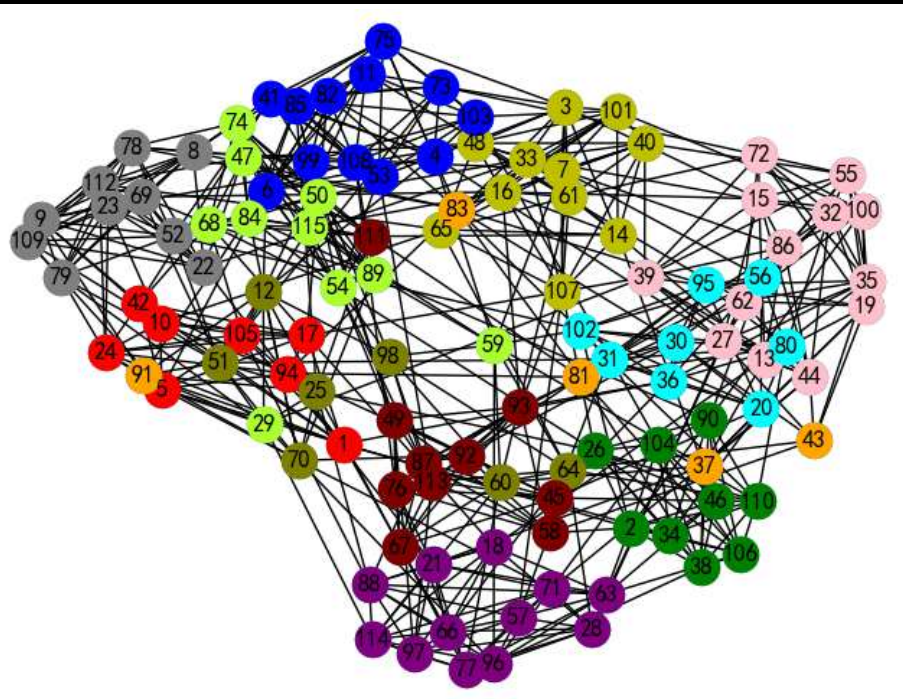

Fig.4 Partition of football data set by DPLPA

It can be seen from fig 3 that although the value of our method is not the best in some data sets, the division result of the DPLPA is the same as the actual community distribution, which can be seen from table 4 and fig 4 . This is mainly because in the process of label dissemination, the probability transition matrix well suppresses the randomness of the dissemination process, so that each update of the node is updated to the label of the same community node as much as possible, making the result of community division More stable and closer to the real community situation. Comparison of $\mathrm{K}$ values of different algorithms on different networks is shown in table 5 and fig 5.

Table $5 \mathrm{~K}$ value comparison of different algorithms in real network

\begin{tabular}{cccccc}
\hline Networks & FN & BGLL & LPA & DPLPA & True K \\
\hline Karate & 3 & 4 & 2 & 2 & 2 \\
Dolphins & 4 & 5 & 4 & 2 & 2 \\
Polbook & 4 & 3 & 4 & 3 & 3 \\
Football & 6 & 10 & 10 & 12 & 12 \\
\hline
\end{tabular}




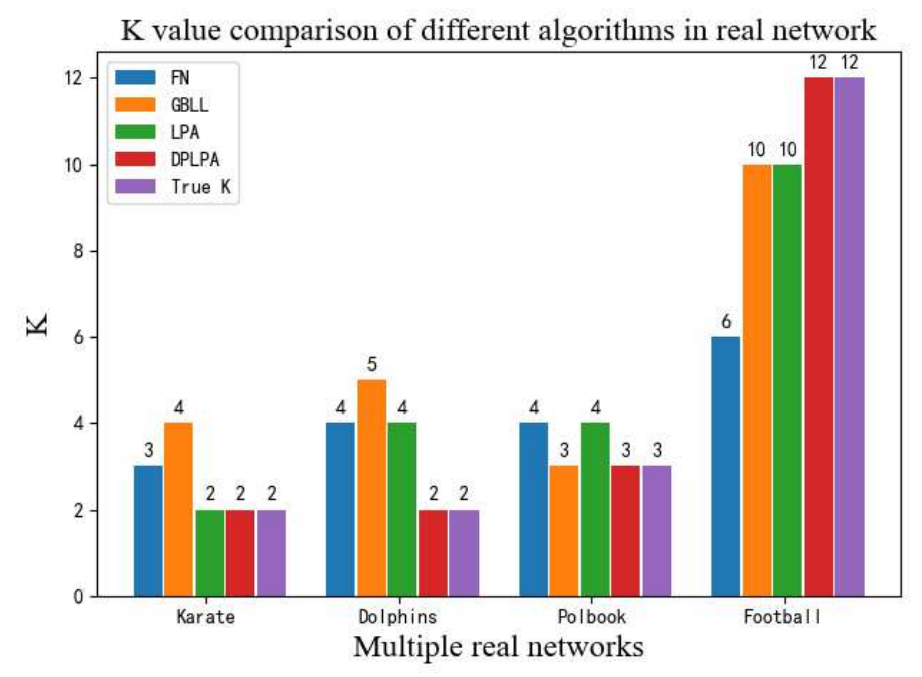

Fig. $5 \mathrm{~K}$ value comparison of multiple algorithms on multiple data sets

In addition, from fig 5, we find that the DPLPA can detect the true number of communities, which is completely consistent with the actual $\mathrm{K}$ value. This is mainly because the DPLPA begins to calculate the local density and distance of nodes through the topology of the network at the very beginning, and selects the number of $\mathrm{K}$ values through a decision graph. Therefore, we do not need to provide the $\mathrm{K}$ value, and the DPLPA has the advantage of detecting the $\mathrm{K}$ value.

In order to better show the experimental results, we use the Karate network and the Dolphins network as case studies to visualize the detected communities. Nodes in the same community are divided by the same color. Fig 6 is the visualization result of DPLPA division of Karate network. Fig 7 is the visualization result of the DPLPA division of the Dolphins network.

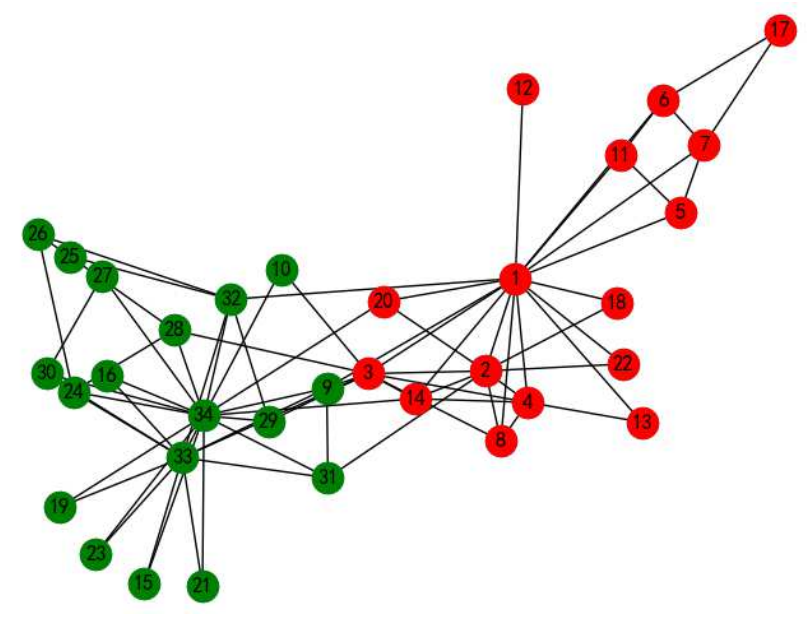


Fig.6 Partition of Karate data set by DPLPA

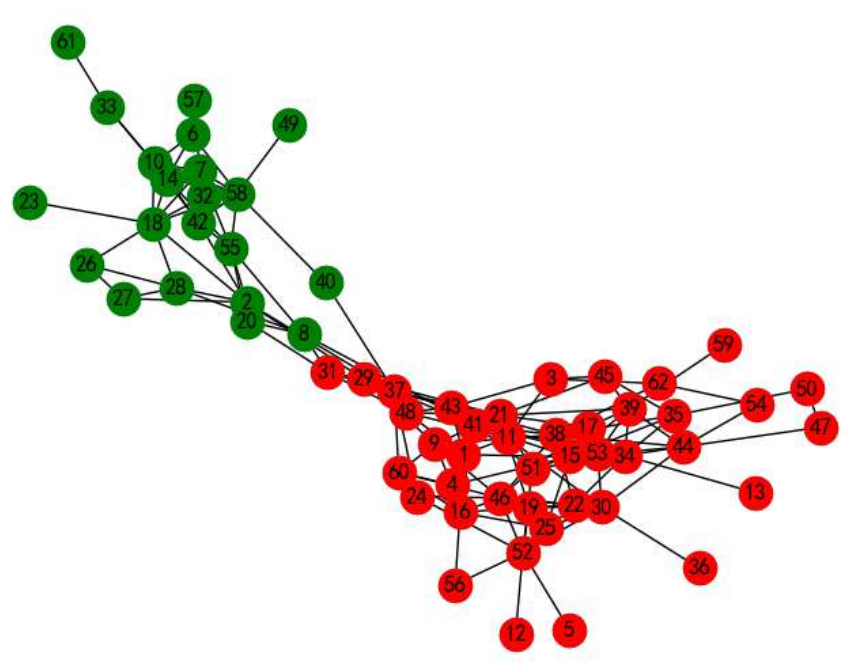

Fig.7 Partition of Dolphins data set by DPLPA

It can be seen from fig 6 that the local density of node 1 and node 34 is the highest, and it can be seen from fig 7 that the local density of node 15 and node 18 is the highest, and these nodes have higher node distance, so it is very reasonable for the DPLPA to select these nodes as $\mathrm{K}$, and the result of the division is completely consistent with the result of the actual community division. Therefore, we believe that the DPLPA is an algorithm that can perform high-quality community detection in real communities. In order to observe the convergence of DPLPA, this paper makes a comparison in multiple data sets, as shown in fig 8 . 

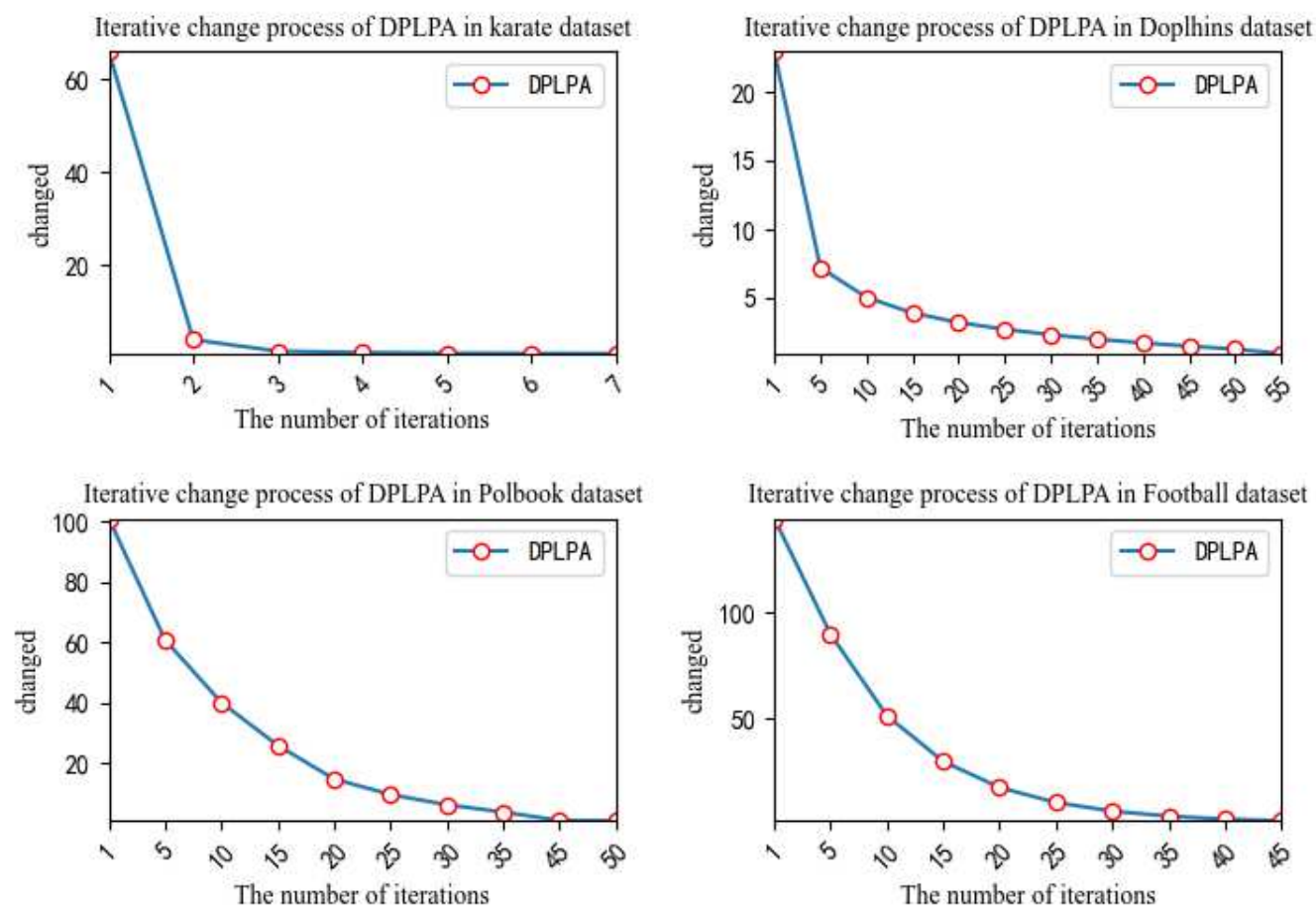

Fig. 8 The changes of node labels in DPLPA algorithm during iteration

Where, $\mathrm{X}$ axis is the number of iterations, and $\mathrm{Y}$ axis is the number of changes during node label iteration. As can be seen from fig 8, in the process of Karate and Doplhins data clustering, the DPLPA has completed the division of most node labels after the first few iterations, and then completed the division of a few nodes. In the process of Polbook and Football data clustering, the labels of most nodes have been partitioned until the 30th iteration. After that, the change curve of node labels becomes flat, indicating that all nodes have completed the label division and the algorithm has converged.

\section{CONCLUDING REMARKS}

In this paper, we propose a DPLPA for complex network community detection. It combines the characteristics of density peak algorithm and can predict the number of communities without a prior condition. It avoids the defects of random label algorithm, such as unstable division and strong randomness, and effectively improves the accuracy of community mining and the stability of the algorithm. In addition, the probability transition matrix is constructed to reduce the number of iterations of label propagation, so that the algorithm has efficient operation time, and finally can quickly find the network community structure. In the test results of the benchmark network and the classical real network, it is found that the proposed algorithm has better stability and accuracy than other advanced algorithms, and the number of communities found is always consistent with the actual number of communities in terms of the predicted $\mathrm{K}$ value. However, there is still room for improvement of the 
algorithm. In future research, we will face large-scale network data and further improve the time complexity of the algorithm. At the same time, dynamic network and overlapping network are also taken as research objects.

\section{Acknowledgement}

This work was supported by Key Science and Technology Program of Henan Province, China(Grant No.162102210168).

\section{REFERENCES}

[1]M. Girvan,M. E. J. Newman. Community Structure in Social and Biological Networks[J]. Proceedings of the National Academy of Sciences of the United States of America,2002,99(12).

[2]Lu Hongchao,Zhu Xiaopeng,Liu Haifeng,Skogerbø Geir,Zhang Jingfen,Zhang Yong,Cai Lun,Zhao Yi,Sun Shiwei,Xu Jingyi,Bu Dongbo, Chen Runsheng. The interactome as a tree--an attempt to visualize the protein-protein interaction network in yeast.[J]. Nucleic acids research,2004,32(16).

[3]M. E. J. Newman. The structure of scientific collaboration networks[J]. Proceedings of the National Academy of Sciences, 2001,98(2).

[4] Fortunato S . Community Detection in Graphs[J]. Physics Reports, 2009, 486(3-5).

[5]Liu, W, Wang, et al. Robust and Scalable Graph-Based Semi-supervised Learning[J]. Proceedings of the IEEE, 2012.

[6] Chong Y , Ding Y, Yan Q, et al. Graph-Based Semi-supervised Learning: A Review[J]. Neurocomputing, 2020.

[7] Nie F , Tian L , Wang R , et al. Multiview Semi-Supervised Learning Model for Image Classification[J]. IEEE Transactions on Knowledge and Data Engineering, 2019, 32(12):1-1.

[8]Alex Rodriguez,Alessandro Laio. Clustering by fast search and find of density peaks[J]. Science,2014,344(6191).

[9]Raghavan Usha Nandini,Albert Réka,Kumara Soundar. Near linear time algorithm to detect community structures in large-scale networks.[J]. Physical review. E, Statistical, nonlinear, and soft matter physics,2007,76(3 Pt 2).

[10]Gergely Tibély,János Kertész. On the equivalence of the label propagation method of community detection and a Potts model approach[J]. Physica A: Statistical Mechanics and its Applications,2008,387(19).

[11]Leung Ian X Y,Hui Pan,Liò Pietro,Crowcroft Jon. Towards real-time community detection in large networks.[J]. Physical review. E, Statistical, nonlinear, and soft matter physics,2009,79(6 Pt 2). 
[12]Barber Michael J,Clark John W. Detecting network communities by propagating labels under constraints.[J]. Physical review. E, Statistical, nonlinear, and soft matter physics,2009,80(2 Pt 2).

[13]X. Liu,T. Murata. Advanced modularity-specialized label propagation algorithm for detecting communities in networks[J]. Physica A: Statistical Mechanics and its Applications,2009,389(7).

[14]Zhao Z X , Wang Y T , Tian J T , et al. A Novel Algorithm for Community Discovery in Social Networks Based on Label Propagation[J]. Journal of Computer Research and Development, 2011, 48.

[15] Xie J , Szymanski B K . LabelRank: A Stabilized Label Propagation Algorithm for Community Detection in Networks[J]. IEEE, 2013.

[16]Zhen Lin,Xiaolin Zheng,Nan Xin,Deren Chen. CK-LPA: Efficient community detection algorithm based on label propagation with community kernel[J]. Physica A: Statistical Mechanics and its Applications,2014,416.

[17]Xian-Kun Zhang,Xue Tian,Ya-Nan Li,Chen Song. Label propagation algorithm based on edge clustering coefficient for community detection in complex networks[J]. International Journal of Modern Physics B,2014,28(30).

[18] Kip F T N ， Welling M. Semi-Supervised Classification with Graph Convolutional Networks[J]. 2017.

[19] Page L , Brin S , Motwani R , et al. The PageRank Citation Ranking: Bringing Order to the Web. Stanford Digital Libraries Working Paper, 1999.

[20]Yakun Li,Hongzhi Wang,Jianzhong Li,Hong Gao. Efficient community detection with additive constrains on large networks[J]. Knowledge-Based Systems,2013,52.

[21]Ma Qianli, Zhang Junhao. A Local Strengthened Multi-label Propagation Algorithm for Community Detection[ J ] . Computer Engineering,2014,4 0 (6) : 171 - 174 .

[22] Zhu X , Ghahramani Z , Lafferty J D . Semi-Supervised Learning Using Gaussian Fields and Harmonic Functions[C]// Machine Learning, Proceedings of the Twentieth International Conference (ICML 2003), August 21-24, 2003, Washington, DC, USA. 2003.

[23]H. Bäcklund, A. Hedblom, and N. Neijman, "DBSCAN: A density-based spatial clustering of application with noise," Linköpings Universitet-ITN, Data Mining TNM033, 2011.

[24]Hong Lu,Qinghua Zhao,Xiaoshuang Sang,Jianfeng Lu. Community Detection in Complex Networks Using Nonnegative Matrix Factorization and Density-Based Clustering Algorithm[J]. Neural Processing Letters,2020(prepublish).

[25]Dillon Martin. Introduction to modern information retrieval: G. Salton and M. McGill. McGraw-Hill, New York (1983). xv + 448 pp., \$32.95 ISBN 0-07-054484-0[J]. Pergamon,1983,19(6).

[26]Newman M E J. Fast algorithm for detecting community structure in networks.[J]. Physical review. E, Statistical, nonlinear, and soft matter physics,2004,69(6 Pt 2).

[27]Vincent D Blondel,Jean-Loup Guillaume,Renaud Lambiotte,Etienne Lefebvre. Fast unfolding of communities in large networks[J]. Journal of Statistical Mechanics: Theory and Experiment,2008,2008(10). 
[28]Estévez Pablo A,Tesmer Michel,Perez Claudio A,Zurada Jacek M. Normalized mutual information feature selection.[J]. IEEE transactions on neural networks,2009,20(2).

[29]Lancichinetti Andrea,Fortunato Santo,Radicchi Filippo. Benchmark graphs for testing community detection algorithms.[J]. Physical review. E, Statistical, nonlinear, and soft matter physics,2008,78(4 Pt 2).

[30]Wayne W. Zachary. An Information Flow Model for Conflict and Fission in Small Groups[J]. Wayne W. Zachary,1977,33(4).

[31]Lusseau David. The emergent properties of a dolphin social network[J]. Proceedings of the Royal Society B: Biological Sciences,2003,270.

[32] Newman M . Modularity and community structure in networks[J]. Proceedings of the National Academy of Sciences of the United States of America, 2006, 103(23):8577-8582. 
Figures

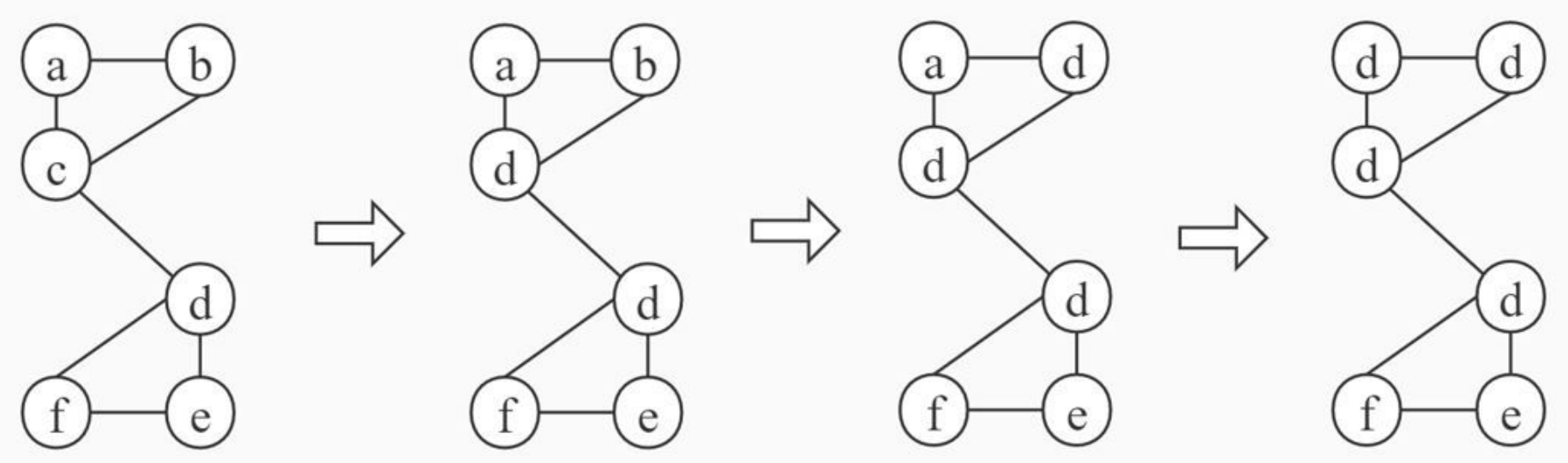

Figure 1

Example of the propagation of an error 


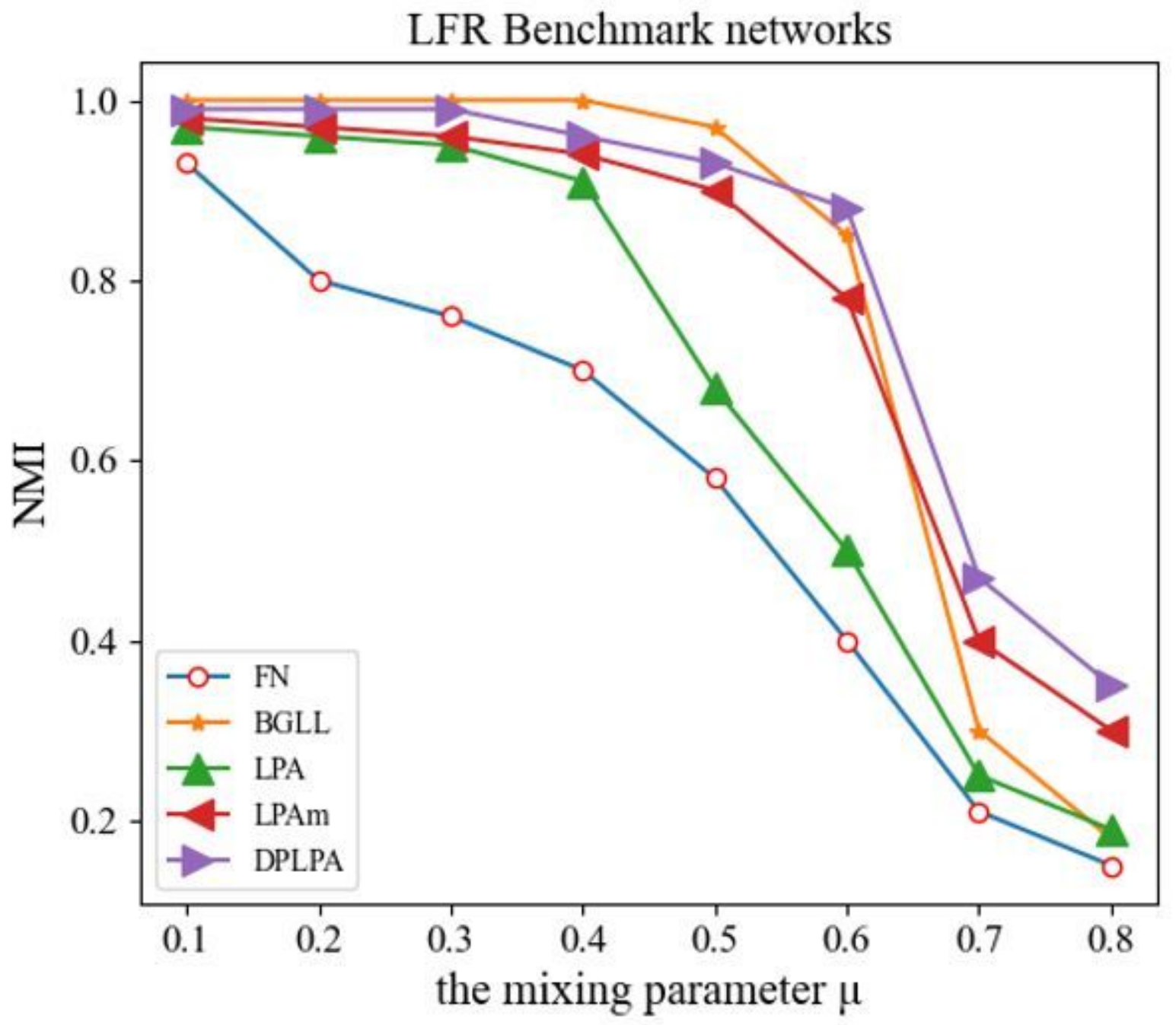

Figure 2

Experimental results on benchmark data set 


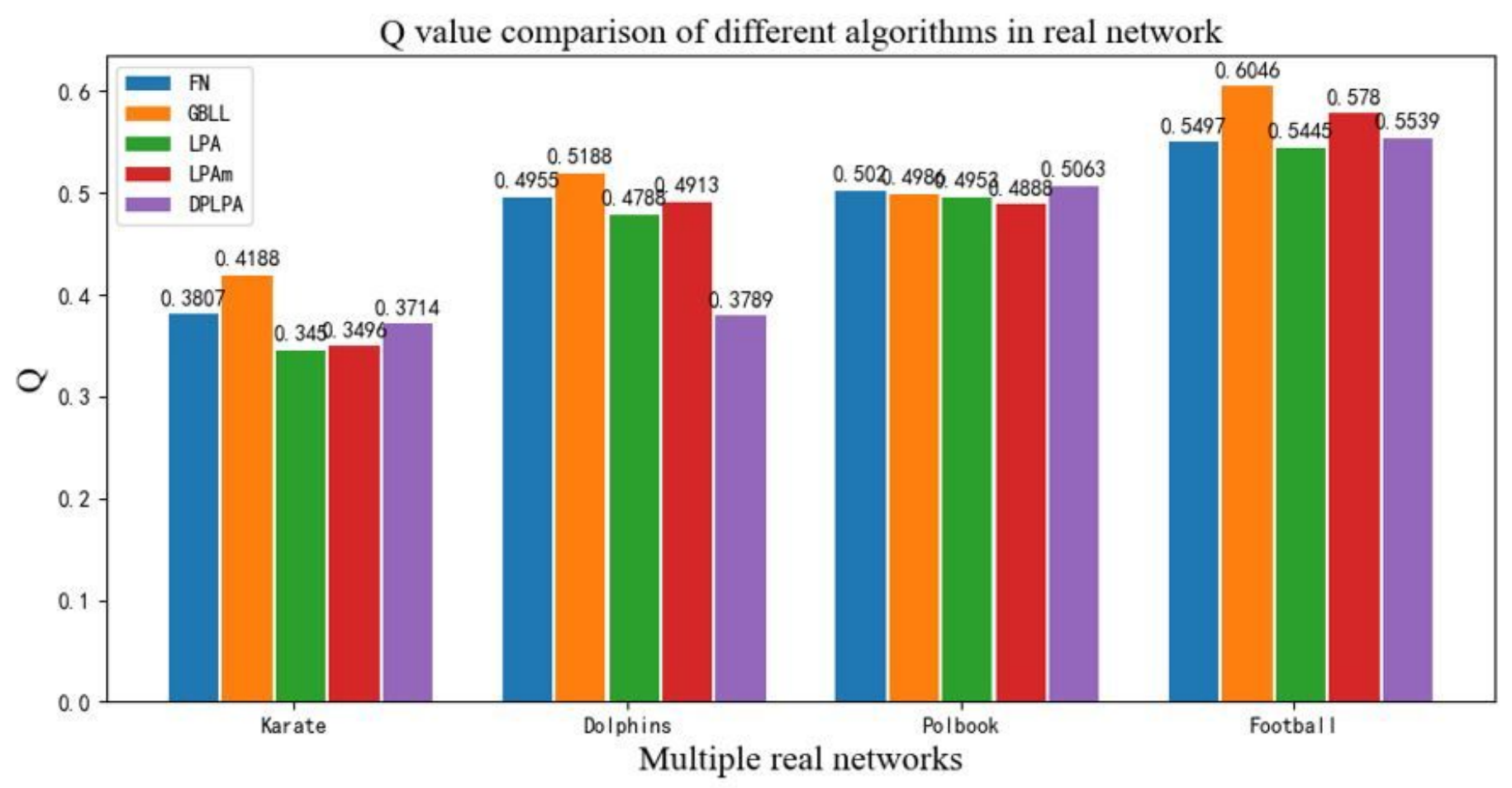

Figure 3

Modularity Q comparison of multiple algorithms on multiple data sets

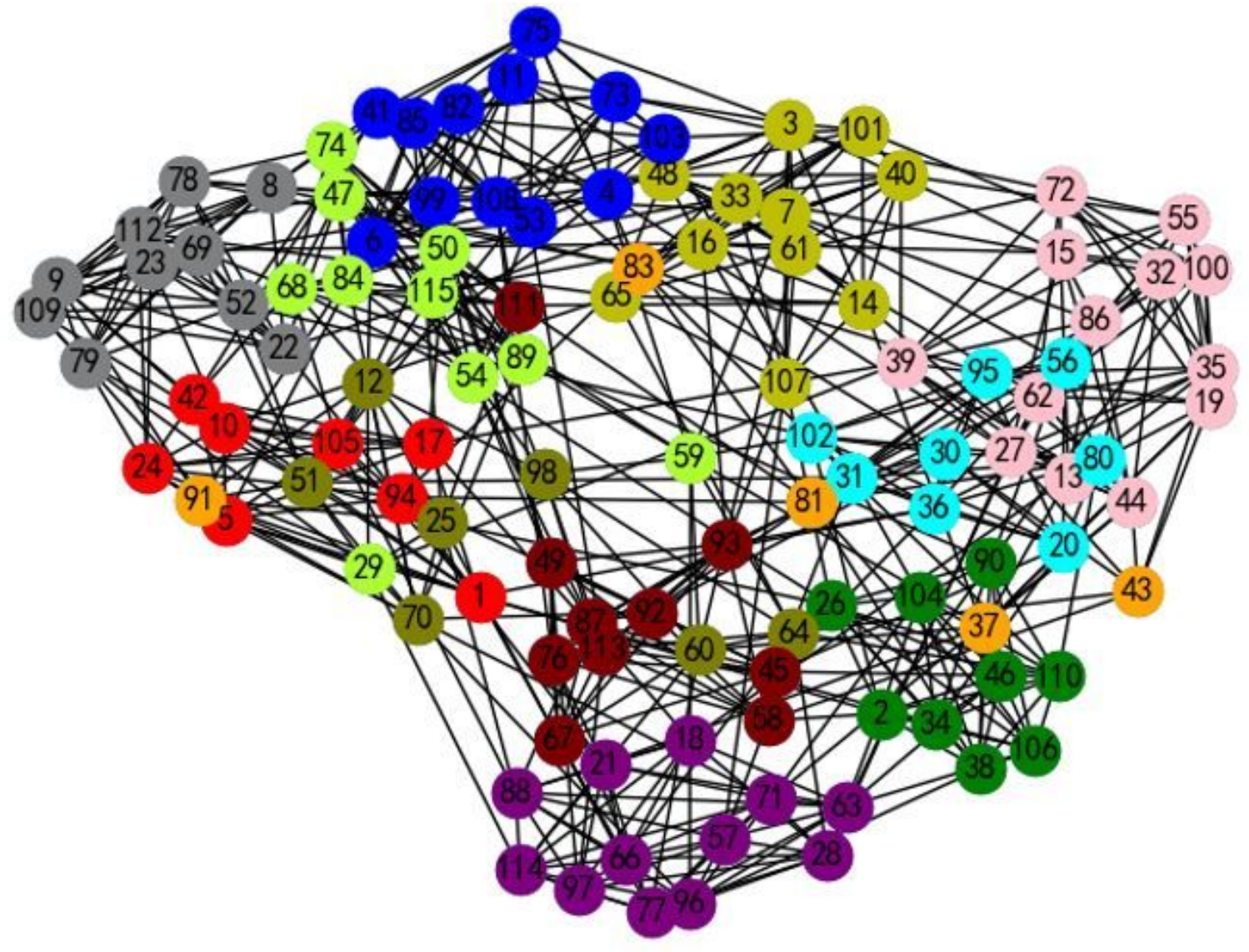


Figure 4

Partition of football data set by DPLPA

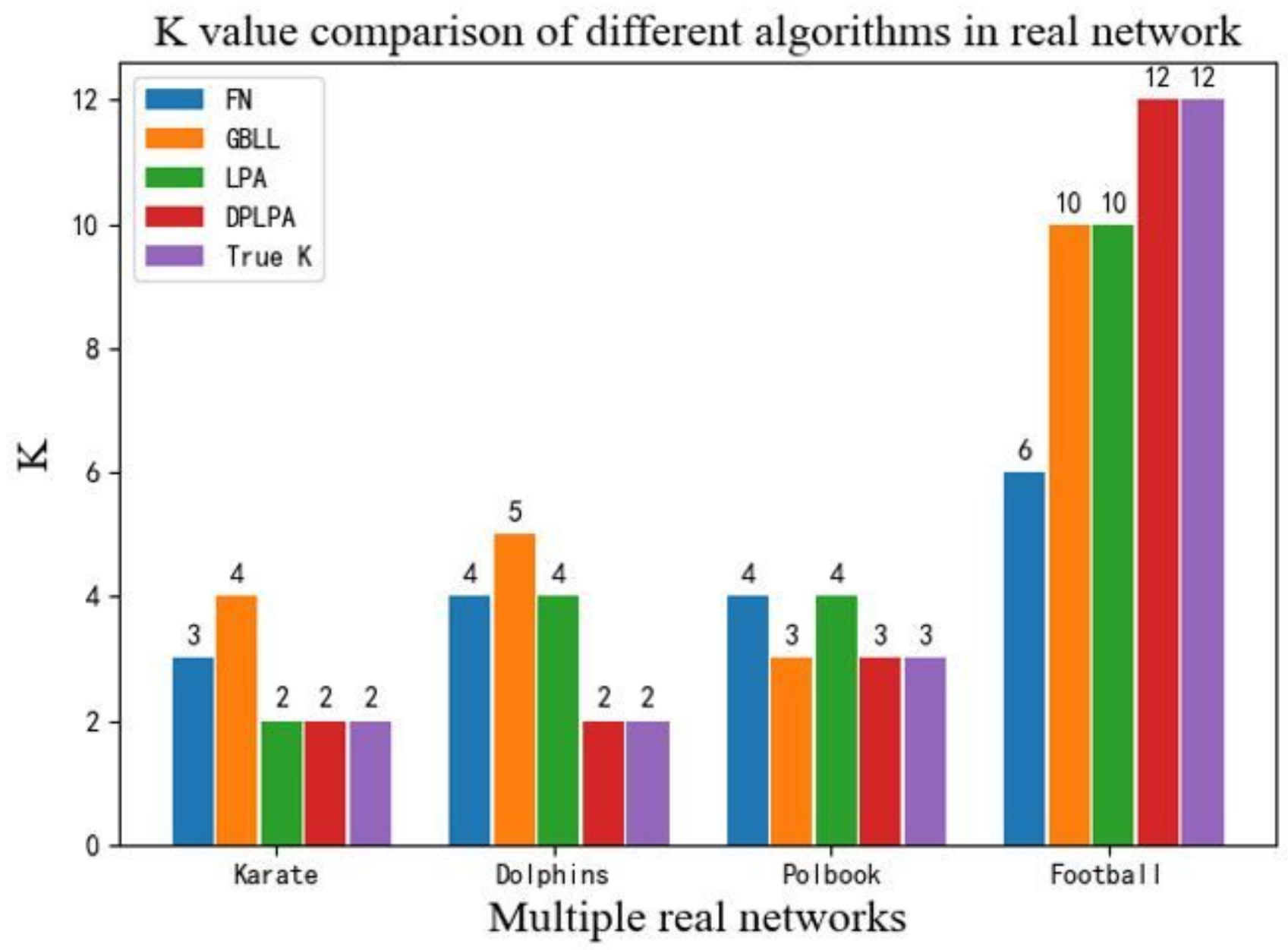

Figure 5

$\mathrm{K}$ value comparison of multiple algorithms on multiple data sets 


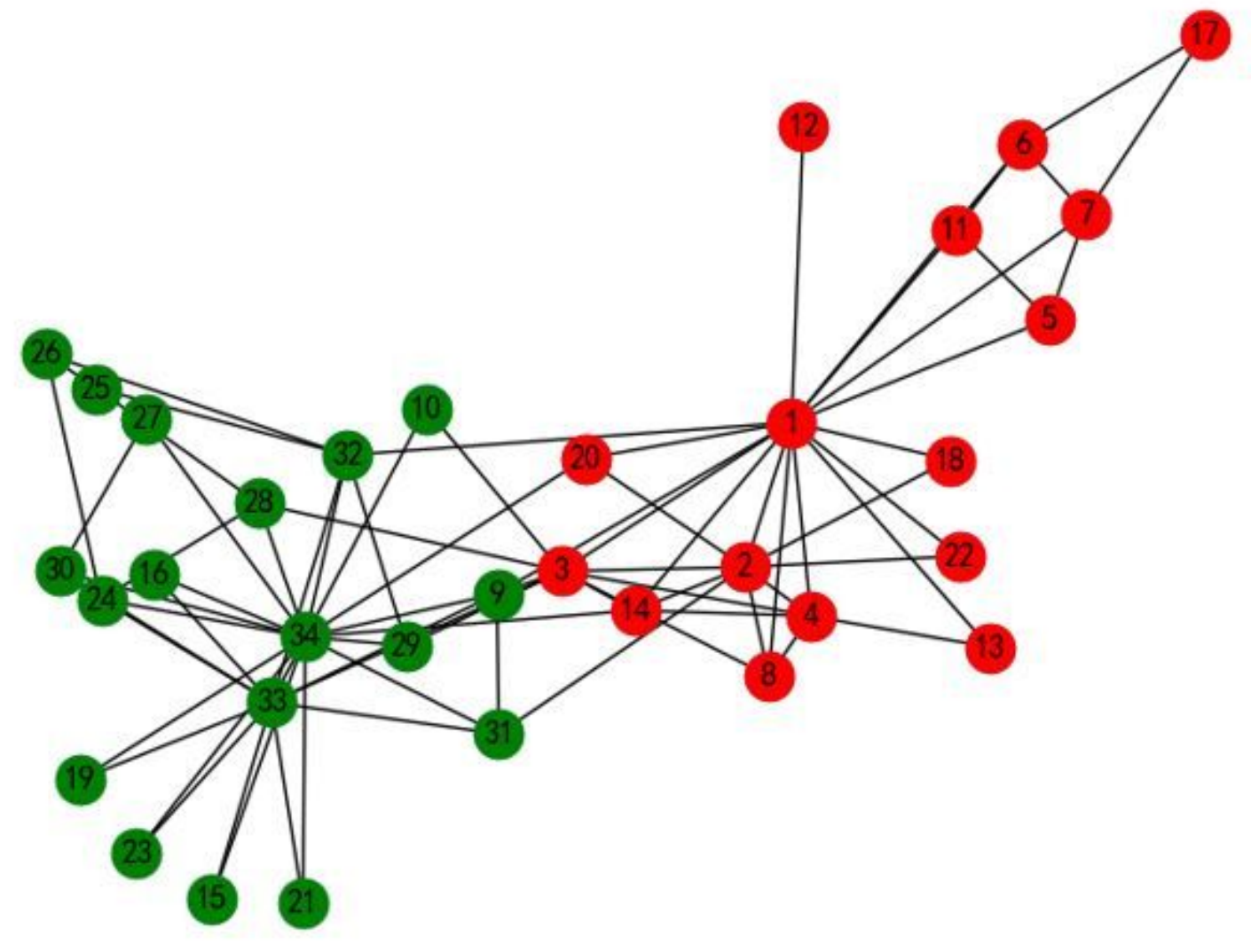

Figure 6

Partition of Karate data set by DPLPA 


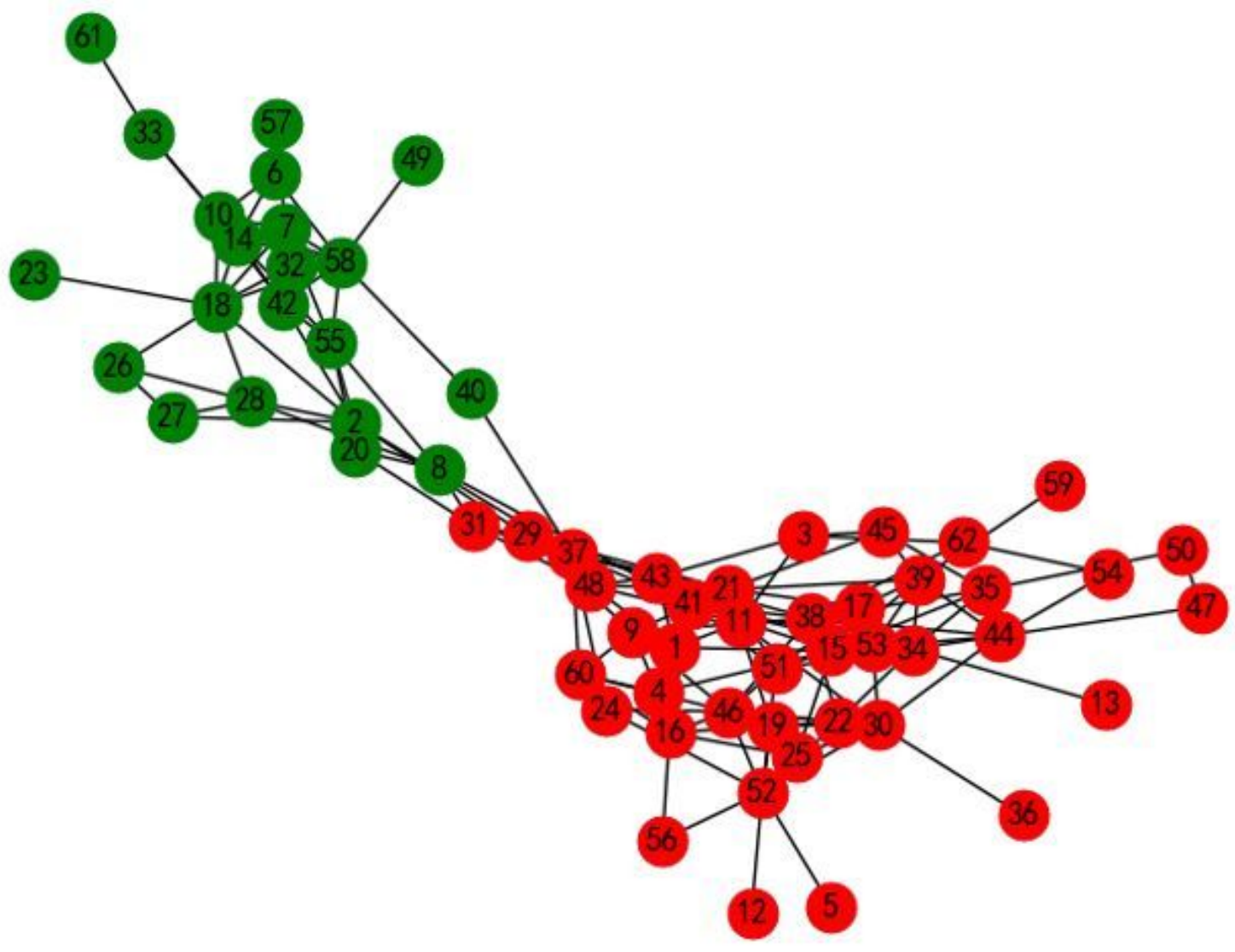

Figure 7

Partition of Dolphins data set by DPLPA 

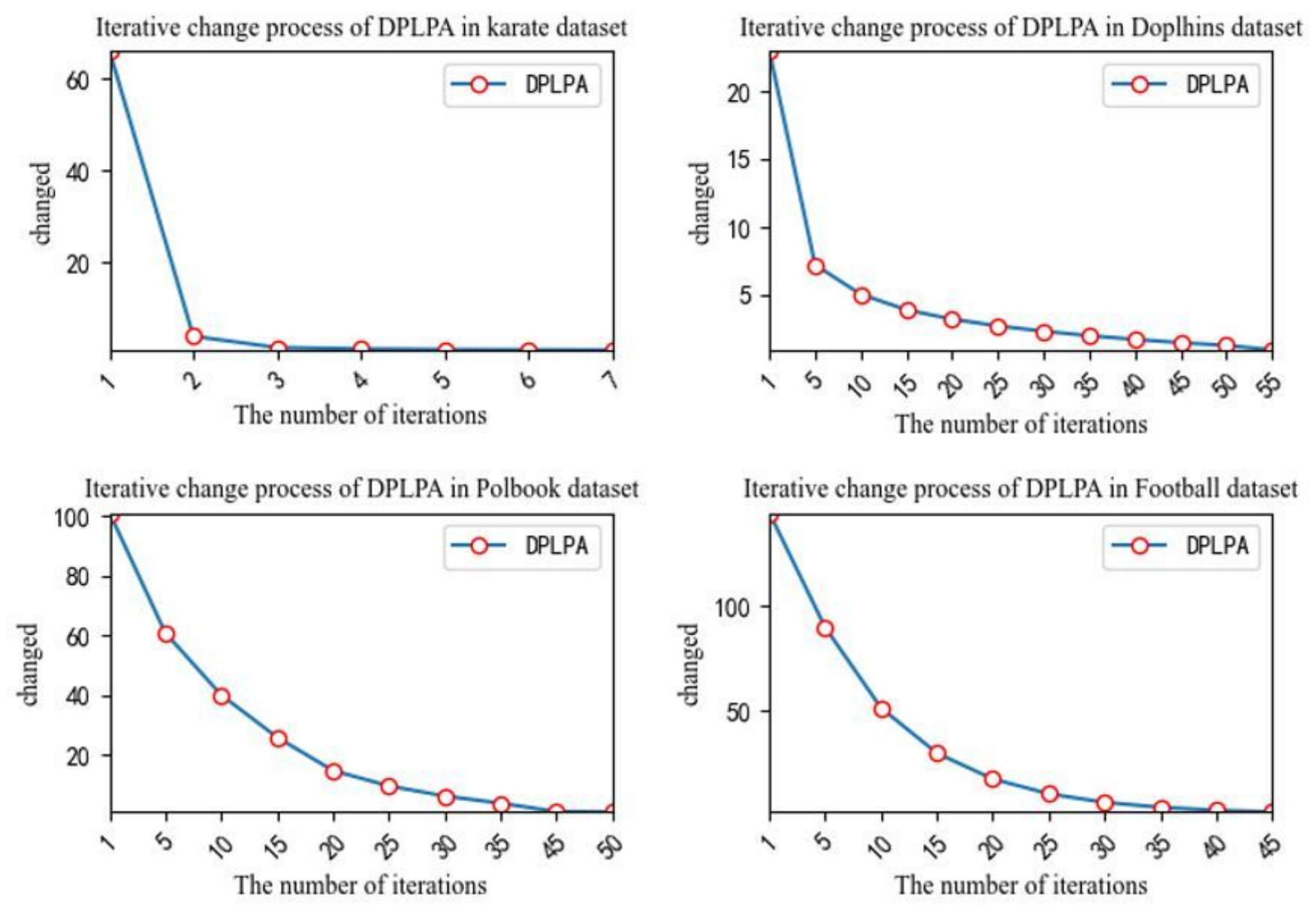

\section{Figure 8}

The changes of node labels in DPLPA algorithm during iteration 